\title{
Functional Brain Hyperactivations Are Linked to an Electrophysiological Measure of Slow Interhemispheric Transfer Time after Pediatric Moderate/Severe Traumatic Brain Injury
}

\author{
Alexander Olsen, ${ }^{1-3}$ Talin Babikian,,4 Emily L. Dennis, ${ }^{1,5-7}$ Monica U. Ellis-Blied, ${ }^{8,9}$ Christopher Giza, ${ }^{4,10,11}$ \\ Sarah DeBoard Marion, ${ }^{12,13}$ Richard Mink, ${ }^{14}$ Jeffrey Johnson, ${ }^{15}$ Christopher J. Babbitt, ${ }^{16}$ \\ Paul M. Thompson, ${ }^{7}$ and Robert F. Asarnow ${ }^{1,17,18}$
}

\begin{abstract}
Increased task-related blood oxygen level dependent (BOLD) activation is commonly observed in functional magnetic resonance imaging (fMRI) studies of moderate/severe traumatic brain injury (msTBI), but the functional relevance of these hyperactivations and how they are linked to more direct measures of neuronal function remain largely unknown. Here, we investigated how working memory load (WML)-dependent BOLD activation was related to an electrophysiological measure of interhemispheric transfer time (IHTT) in a sample of 18 msTBI patients and 26 demographically matched controls from the UCLA RAPBI (Recovery after Pediatric Brain Injury) study. In the context of highly similar fMRI task performance, a subgroup of TBI patients with slow IHTT had greater BOLD activation with higher WML than both healthy control children and a subgroup of msTBI patients with normal IHTT. Slower IHTT treated as a continuous variable was also associated with BOLD hyperactivation in the full TBI sample and in controls. Higher WML-dependent BOLD activation was related to better performance on a clinical cognitive performance index, an association that was more pronounced within the patient group with slow IHTT. Our previous work has shown that a subgroup of children with slow IHTT after pediatric msTBI has increased risk for poor white matter organization, long-term neurodegeneration, and poor cognitive outcome. BOLD hyperactivations after msTBI may reflect neuronal compensatory processes supporting higher-order capacity demanding cognitive functions in the context of inefficient neuronal transfer of information. The link between BOLD hyperactivations and slow IHTT adds to the multi-modal validation of this electrophysiological measure as a promising biomarker.
\end{abstract}

Keywords: brain reserve; EEG; functional magnetic resonance imaging; interhemispheric transfer time; traumatic brain injury

\footnotetext{
${ }^{1}$ Department of Psychiatry and Biobehavioral Sciences, Semel Institute for Neuroscience and Human Behavior, ${ }^{11}$ Departments of Pediatrics and Neurosurgery, David Geffen School of Medicine at UCLA, ${ }^{17}$ Department of Psychology, and ${ }^{18}$ Brain Research Institute, UCLA, Los Angeles, California.

${ }^{2}$ Department of Psychology, NTNU, Norwegian University of Science and Technology, Trondheim, Norway.

${ }^{3}$ Department of Physical Medicine and Rehabilitation, St. Olav's Hospital, Trondheim University Hospital, Trondheim, Norway.

${ }^{4}$ UCLA Steve Tisch BrainSPORT Program, Los Angeles, California.

${ }^{5}$ Psychiatry Neuroimaging Laboratory, Brigham \& Women's Hospital, Boston, Massachusetts.

${ }^{6}$ Stanford Neurodevelopment, Affect, and Psychopathology Laboratory, Stanford, California.

${ }^{7}$ Imaging Genetics Center, Stevens Neuroimaging \& Informatics Institute, Keck School of Medicine of USC, Marina del Rey, California.

${ }^{8}$ Fuller Theological Seminary School of Psychology, Pasadena, California.

${ }^{9}$ Loma Linda VA Healthcare System, Loma Linda, California.

${ }^{10}$ UCLA Mattel Children's Hospital, Los Angeles, California.

${ }^{12}$ Department of Psychology, Northwest Nazarene University, Nampa, Idaho.

${ }^{13}$ Elk's Rehabilitation Hospital, St. Luke's Health System, Boise, Idaho.

${ }^{14}$ Department of Pediatrics, Harbor-UCLA Medical Center and Los Angeles Biomedical Research Institute, Torrance, California.

${ }^{15}$ Department of Pediatrics LAC+USC Medical Center and Keck School of Medicine, Los Angeles, California.

${ }^{16}$ Miller Women's and Children's Hospital of Long Beach, Long Beach, California.
}

(c) Alexander Olsen et al., 2019; Published by Mary Ann Liebert, Inc. This Open Access article is distributed under the terms of the Creative Commons License (http://creativecommons.org/licenses/by/4.0), which permits unrestricted use, distribution, and reproduction in any medium, provided the original work is properly credited. 


\section{Introduction}

I

NCREASED TASK-RELATED blood oxygen level dependent (BOLD) activation is the most common observation in functional magnetic resonance imaging (fMRI) studies of moderate-to-severe traumatic brain injury (msTBI). In general, BOLD activation typically increases with higher task load, as well as with other extrinsic factors (e.g., aging, sleep-wake disturbance, brain injury, and disease) that are associated with increased cerebral metabolic demand. ${ }^{1,2}$ In msTBI, BOLD hyperactivations are observed across different tasks and may represent injury-related compensatory mechanisms reflecting increased effort required to maintain task performance, inefficient neuronal processing, and/or merely changes in neurovascular coupling after injury. ${ }^{1,3-7}$ Task-elicited hyperactivations are the most consistent finding when fMRI task performance is maintained similarly between patients and controls, but some studies have also shown instances of hypoactivations in pediatric msTBI. ${ }^{8,9}$

Interpretation of fMRI findings in pediatric msTBI is complicated by the heterogeneous nature of msTBI pathology, small sample sizes, and the fact that few studies have linked BOLD alterations to clinical variables (e.g., Glasgow Coma Scale [GCS] score, time post-injury, and neuropsychological test measures), and none have investigated the relationship to more direct measures of neuronal functioning. Extant studies do not provide a clear understanding of the functional and clinical significance of BOLD alterations after msTBI in children and adolescents. To further increase our understanding of BOLD alterations after msTBI, the role of these alterations should not only be further validated by traditional neurocognitive and clinical measures, but also more direct measures of neuronal functioning.

Our previous work identified a subgroup of msTBI patients with slow interhemispheric transfer time (IHTT; a scalp visual eventrelated potential [ERP] measure of corpus callosum function). ${ }^{10}$ TBI patients with slow IHTT had poor white matter organization, long-term neurodegeneration, and poor cognitive outcome ${ }^{10-13}$ compared to msTBI patients with normal IHTT. Slow IHTT is therefore a promising electrophysiological biomarker in pediatric msTBI. ${ }^{14}$ Examining the relation between the electrophysiological IHTT measure and BOLD alterations will provide a significant step toward a more substantial understanding of the effect of msTBI on brain function, including determining whether alterations in the BOLD signal after msTBI reflect functional neuronal changes, and not just potential changes in neurovascular coupling.

Slow IHTT is a basic measure of neuronal efficiency that is likely to affect processing across several cognitive domains given that it is calculated based on the visual N1 component, ${ }^{4}$ which appears before ERPs related to domain-specific higher cognitive operations (e.g., P3). ${ }^{5}$ Slowed processing on a behavioral level has been suggested to partly explain BOLD hyperactivations after adult msTBI, but whether this actually represents recruitment of latent support mechanisms attributable to inefficient neuronal processing remains to be determined. ${ }^{6,7}$ Computationally demanding higherorder cognitive processes, such as working memory, are particularly dependent on coordinated processing in both hemispheres and, consequently, sensitive to altered IHTT. ${ }^{8}$ A clear advantage of using working memory tasks in fMRI research is that task load can be parametrically manipulated, ${ }^{9}$ while still preserving the opportunity to adjust for between-subject task performance by exclusively including correct trials when modeling the BOLD response. ${ }^{10}$ This provides a powerful approach to evaluating the consequences of higher cerebral demand ${ }^{11,12}$ as reflected by increased working memory load (WML)-dependent BOLD activation.
Taken together, both increased WML and slow behavioral response time after msTBI seem to represent increased demands on the brain, providing a need for recruiting extra neuronal resources to uphold task performance. After pediatric msTBI, it is likely that the consequences of slow IHTT and poor white matter organization become more evident with higher WML as the need to transfer and integrate information across the brain increases. In the context of accurate task performance, individuals with longer IHTT would therefore be expected to show BOLD hyperactivation as a function of higher WML.

Here we investigated spatial working memory task-related BOLD activations after pediatric msTBI in a subsample from the UCLA RAPBI (Recovery After Pediatric Brain Injury) study ${ }^{10}$ that had both fMRI and IHTT data available. Both higher WML and suboptimal neuronal transfer of information (operationalized as slow IHTT) as indices of increased cerebral demand ${ }^{1,2}$ were expected to be associated with increased BOLD activation. Specifically, we hypothesized that pediatric msTBI patients with slow IHTT would demonstrate a greater increase in BOLD activation as WML increased compared to msTBI individuals with normal IHTT and a healthy control group, possibly reflecting compensatory mechanisms. The external validity of BOLD alterations was further evaluated in the full msTBI sample by investigating associations with injury-related variables and a clinical measure of neurocognitive function. ${ }^{20}$

\section{Methods \\ Participants}

TBI patients were recruited from four different pediatric intensive care units located in level 1 and 2 trauma centers in Los Angeles County. Patients were included if they experienced a moderate-to-severe non-penetrating TBI, with intake or postresuscitation GCS between 3 and 12 (or higher if there were confirmed abnormalities on clinical imaging), if they were between 8 and 18 years of age at the time of injury, had normal visual acuity or corrected vision with contact lenses or eyeglasses, and had sufficient English skills to understand instructions and participate in neurocognitive testing presuming English competence.

Participants were excluded if they had previous head injury, motor deficits precluding them from participating in the test protocols, or other significant pre-trauma history of neurological, developmental, or psychiatric disorders. Healthy control participants were matched on age, sex, and educational level to the TBI patients and were recruited from the community through flyers, magazines, and school postings. With the exception of criteria for TBI injury severity, healthy control participants met the same inclusion and exclusion criteria as TBI patients. Finally, participants were excluded from the MRI part of the study if they were not eligible for MRI (e.g., because of having metal implants that were not MRI compatible). The study was approved by the University of California, Los Angeles (UCLA) institutional review board and the institutional review boards of each site from which patients were recruited.

The study design has been described in greater detail elsewhere. ${ }^{10}$ Fifty patients were included in the overall study. However, because fMRI data collection was initiated late in the project, a total of 18 (4 female) patients had both task-fMRI and IHTT data collected at the same time point of sufficient quality to be included in this study. A group of 26 (11 female) demographically matched controls, with both task-fMRI and IHTT data, was also included. The TBI group was further divided into subgroups based on IHTT as previously described. ${ }^{10}$ Briefly, electroencephalography was recorded using a BIOSEMI system (BioSemi, Amsterdam, the Netherlands; sampling rate $=512 \mathrm{~Hz}$, low-pass filter $=40 \mathrm{~Hz}$, high- 
pass filter $=0.16 \mathrm{~Hz}$, bandwidth $[3 \mathrm{~dB}]=134 \mathrm{~Hz}$ ) while participants performed a pattern matching task with bilateral field advantage. Stimuli for each trial were presented randomly and pairwise in two of four visual fields (upper and lower; right and left), creating four bilateral and two unilateral conditions.

Participants were asked to indicate whether the two stimuli (patterns) constituted a "match" or "non-match" by pressing a keyboard. The responding hand (right or left) was alternated in eight blocks of 97 trials each. ERPs time-locked to the stimulus presentation were calculated from parietal (P3/P4) and occipital (O1/O2) electrodes. The peak latency (in milliseconds) of the early visual N1 (typically observed $\sim 150-200 \mathrm{~ms}$ post-stimulus presentation) component was determined and averaged for the right and left hemisphere. An overall IHTT was calculated by averaging left to right and right to left differences (IHTTs) based on data from the unilateral conditions. Longer IHTT indicates slower transfer of visual information between the hemispheres, which is a basic and robust measure of neuronal efficiency, given that the N1 component reflects early visual registration occurring before any involvement of domain-specific higher cognitive operations, such as those reflected in cognitive ERPs, such as the P3. ${ }^{5}$

In the overall study, IHTT had a skewed distribution in the msTBI patients, with around half of the group having scores within 1.5 standard deviations (SDs) of the normal range, as calculated based on data from the healthy control group. ${ }^{4}$ The balance of the TBI group had slow IHTT outside the normal range ( $>1.5$ SDs below the mean of the control group). The bimodal properties of the data therefore supported a meaningful cutoff for defining slow IHTT as being above 1.5 SDs of the mean of healthy controls. ${ }^{4}$ This cutoff has been successfully used in our earlier studies showing that TBI patients with slow IHTT had poor white matter organization, long-term neurodegeneration, and poor cognitive outcome. ${ }^{4,13-15}$ In the current study, subjects with an IHTT score of $>1.5$ SDs ( $\geq 18 \mathrm{~ms}$ ) from the mean of the entire healthy control group that was included in the overall study ${ }^{10}$ were allocated to the "TBI slow" group ( $n=7,1$ female), and the remaining patients were included in the "TBI normal" group ( $n=11,3$ female). Demographic and clinical data for the groups are presented in Table 1.

\section{Clinical measure of neurocognitive function}

A previously validated cognitive performance index (CPI) was included as a clinical measure of neurocognitive function. ${ }^{20}$ This index combined standardized scores from tests measuring verbal memory, psychomotor skills, working memory, and inhibition/ set-switching into a single psychometrically sound summary score representing overall cognitive efficiency. ${ }^{20}$

\section{Analyses of demographic and clinical measures}

Means, SDs, and $95 \%$ confidence intervals (CIs) of the means were calculated (Table 1). For measures where all groups had data, separate analyses of variance (ANOVAs) with group (TBI slow, TBI normal, and controls) as a between-subject factor and each dependent variable as a within-group factor were performed. Statistically significant main effects were followed up by pair-wise between-group contrasts. For TBI-related measures, pair-wise comparisons using an independent $t$-test were applied. The acceptance level for statistically significant results was set to $p<0.05$, and partial eta squared $\left(\eta \rho^{2}\right)$ was calculated as a measure of effect size.

IBM SPSS Statistics Version 24 was used for statistical analysis of demographic, clinical, and behavioral data.

\section{Spatial working memory task}

BOLD fMRI was acquired during performance of a parametric spatial working memory task. Before each trial, four squares appeared on the screen, indicating the spatial locations where stimuli could appear. After each trial, these squares were replaced with triangles, indicating that the participant should start responding. For each trial, one to five pictures (items) of fruits and vegetables were sequentially presented for $800 \mathrm{~ms}$ within the four possible different locations on the screen. Interstimulus time was $200 \mathrm{~ms}$, and intertrial time was $1500 \mathrm{~ms}$ (from the last response to the beginning of a new sequence). Subjects were instructed to attend to the four positions on the screen and reproduce the order of the presentation (not what pictures were presented) by using a response box with four buttons (Current Designs, Winona, MN) corresponding to the respective spatial locations. Because several similar objects were presented (e.g., 25 different apples), respondents were not able to associate a unique word with each stimulus, thereby minimizing verbal mediation as a working memory strategy.

Participants were asked to respond as quickly and accurately as possible. All participants completed a practice session before the actual fMRI using a mock setup before their scan to ensure understanding of task instructions.

Task demands were manipulated in a parametric manner giving four trial types with different WML: baseline (one item), WML 3

Table 1. Demographics and Clinical Measures

\begin{tabular}{|c|c|c|c|c|c|}
\hline Variable & F-statistics & Group & $\mathrm{n}$ & Mean (SD) & $95 \%$ CI of means \\
\hline \multirow[t]{3}{*}{ Age } & \multirow[t]{3}{*}{$F_{(2,41)}=0.979, p=0.384, \eta 2=0.046$} & Controls & 26 & $15.78(3.12)$ & $14.64,16.92$ \\
\hline & & TBI normal & 11 & $14.33(2.67)$ & $12.58,16.09$ \\
\hline & & TBI slow & 7 & $15.28(2.09)$ & $13.08,17.48$ \\
\hline \multirow[t]{3}{*}{ IHTT average (msec) } & \multirow{3}{*}{$F_{(2,41)}=36.70, p<0.001, \eta 2=0.642$} & Controls & 26 & $7.58(5.78)^{*}$ & $5.43,9.73$ \\
\hline & & TBI normal & 11 & $4.50(3.75)^{\dagger}$ & $1.20,7.81$ \\
\hline & & TBI slow & 7 & $25.50(7.58)^{* \dagger}$ & $21.36,29.64$ \\
\hline \multirow{3}{*}{$\begin{array}{l}\text { Cognitive Performance Index } \\
\text { (standard score) }\end{array}$} & \multirow[t]{3}{*}{$F_{(2,41)}=9.109, p=0.001, \eta 2=0.308$} & Controls & 26 & $106.24(9.77)^{* \dagger}$ & $102.17,110.30$ \\
\hline & & TBI normal & 11 & $93.13(11.54)^{\dagger}$ & $86.88,99.38$ \\
\hline & & TBI slow & 7 & $92.32(10.00)^{*}$ & $84.49,100.16$ \\
\hline \multirow[t]{2}{*}{ GCS at admission } & \multirow[t]{2}{*}{-} & TBI normal & 11 & $9.00(3.35)$ & $6.89,11.11$ \\
\hline & & TBI slow & 7 & $9.00(3.21)$ & $6.36,11.64$ \\
\hline \multirow[t]{2}{*}{ Time post-injury (weeks) } & \multirow[t]{2}{*}{-} & TBI normal & 11 & $38.08(31.64)$ & $19.51,58.18$ \\
\hline & & TBI slow & 7 & $38.85(29.38)$ & $12.85,61.32$ \\
\hline
\end{tabular}

Footnote symbols $(*$ or $\dagger)=$ pair-wise statistically significant difference, $p<0.05$.

IHTT, interhemispheric transfer time; TBI, traumatic brain injury; GCS, Glasgow Coma Scale; SD, standard deviation; CI, confidence interval; ANOVA, analysis of variance; $\eta 2$, partial eta squared. 
(three items), WML 4 (four items), and WML 5 (five items). The task consisted of a total of 128 trials divided into 32 trials of each trial type, divided into four equally long time epochs that were balanced with regard to WML and intratrial sequence order to avoid potential order effects. Stimuli were presented through a head-coil mounted mirror system and an in-house custom-built MRI compatible video projector (Staglin Center, UCLA). The task design was implemented using the PsychToolbox in Matlab (The MathWorks, Inc., Natick, MA). A log file containing behavioral data was stored, and response time and the number of correct responses were computed separately for each WML as measures of accuracy and response speed.

\section{Analysis of functional magnetic resonance imaging behavioral data}

Means and SDs for each behavioral measure were calculated separately for the respective groups (Table 2). To investigate within- and between-group effects of WML, separate repeatedmeasures ANOVAs with group (TBI slow, TBI normal, and controls) as the between-subject factor and WML (baseline, WML 3, WML 4, and WML 5) as the within-group factor were performed. Mauchley's test was used to investigate the assumption of sphericity of the data, and a Greenhouse-Geisser correction was used if this assumption was violated. Statistically significant main effects were followed up by pair-wise between-group contrasts. Subsequent polynomial trend analyses were used to further evaluate the expected linear WML effects. The acceptance level for statistically significant results was set to $p<0.05$. Partial eta squared $\left(\eta \rho^{2}\right)$ was calculated as a measure of effect size.

\section{Scan acquisition}

All scans were performed on a Siemens Trio with a 12-channel Head Matrix Coil (Siemens AG, Erlangen, Germany). Foam pads around the subjects' heads were used to reduce head motion. During the parametric spatial working memory task, T2*-weighted BOLD fMRI was acquired utilizing an echo-planar imaging pulse sequence (repetition time $=2400 \mathrm{~ms}$, echo time $=35 \mathrm{~ms}$, field of view $=244 \mathrm{~mm}$, matrix $=80 \times 80$, slice thickness $=3 \mathrm{~mm}$, number of slices $=40$, giving an in-plane resolution of $3 \times 3 \mathrm{~mm}$ ). A T1-weighted three-dimensional magnetization-prepared rapid gradient-echo (MPRAGE) volume was acquired for anatomical reference.

\section{Analysis of magnetic resonance imaging data}

All analyses were performed using the FMRIB's Software Library (FSL) toolbox (version 5.0.7; FMRIB Centre, Oxford, UK). Non-brain structures were removed with BET. ${ }^{21}$ The fMRI data were motion corrected with MCFLIRT, ${ }^{22}$ smoothed (Gaussian kernel full width at half maximum, $6 \mathrm{~mm}$ ), grand mean normalized, high-pass temporal filtered $(50 \mathrm{sec})$, and linearly registered to native high-resolution space (T1 MPRAGE), using a 7 degrees of freedom spatial transformation. ${ }^{22,23}$ A transformation matrix was created by registration of the high-resolution T1 MPRAGE to a 2-mm Montreal Neurological Institute (MNI) standard template using 12 degrees of freedom, and fMRI data were subsequently transformed into standard MNI space by applying the same transformation matrix.

BOLD activation was modeled using a general linear model, and the hemodynamic response function was convolved with a standard Gamma variate. A main contrast was computed to model the linear increase of BOLD activation as a function of WML (baseline, WML 3, WML 4, and WML 5). It is important for the validity of fMRI studies comparing patient and healthy control groups that task performance in the included contrasts is highly similar. ${ }^{19}$ Therefore, only trials with correct performance were included. First, all fMRI contrasts were computed for each participant using a fixed-effects model. Then, mixed-effects models with automatic outlier deweighting were used to create statistical parametric maps (SPMs) and to investigate group differences and within-group associations. The main analysis focused on investigating differences between the predefined IHTT groups: TBI slow, TBI normal, and healthy controls.

To substantiate our findings, we evaluated the clinical and functional relevance of the BOLD activations by performing within-group analyses for the full TBI sample, evaluating associations with age, IHTT (as a continuous variable), the CPI, injury severity (GCS score at admission), and time post-injury (number of weeks). In the control group, we evaluated associations with age, IHTT, and the clinical neurocognitive performance index.

All SPMs were corrected for multiple comparisons by applying a cluster threshold of $Z>2.3$ and a cluster significance threshold of $p<0.05$. Peak $Z$-values with up to five local maxima and the size of clusters (number of voxels) in standard $2 \times 2 \times 2 \mathrm{~mm}$ MNI space were extracted. For anatomical denotation, visual inspection and the Harvard Oxford cortical and subcortical structural brain atlases as incorporated in FSL were applied.

\section{Results}

\section{Demographic and clinical data}

Results are presented in Table 1 . There were no statistically significant differences in age between the TBI slow, TBI normal, and control groups. As expected, the TBI slow group had slower IHTT than both the TBI normal group and controls, but there was no statistically significant difference in IHTT between the TBI normal group and controls. Both TBI groups performed worse than controls on the clinical neurocognitive performance index, but did not differ significantly from each other. There was also no

Table 2. Working Memory fMri Task Performance

\begin{tabular}{llrccrr}
\hline Variable & Group & $\mathrm{n}$ & $\begin{array}{c}\text { Baseline } \\
\text { mean }(S D)\end{array}$ & $\begin{array}{c}\text { WML 3 } \\
\text { mean }(S D)\end{array}$ & $\begin{array}{c}\text { WML 4 } \\
\text { mean }(S D)\end{array}$ & $\begin{array}{c}\text { WML 5 } \\
\text { mean }(S D)\end{array}$ \\
\hline Accuracy (correct responses) & Controls & 26 & $31.19(1.88)$ & $26.92(4.87)$ & $24.65(5.75)$ & $22.00(7.38)$ \\
& TBI normal & 11 & $30.81(1.47)$ & $24.64(5.97)$ & $22.73(6.08)$ & $17.91(7.11)$ \\
& TBI slow & 7 & $31.14(1.21)$ & $26.57(4.69)$ & $25.29(3.15)$ & $21.71(4.79)$ \\
Response speed (seconds) & Controls & 26 & $0.814(.245)$ & $1.637(0.378)^{*}$ & $2.116(0.533) *$ & $2.784(1.109)$ \\
& TBI normal & 11 & $1.113(.594)$ & $2.156(0.523)^{*}$ & $2.766(0.730)^{*}$ & $3.256(0.772)$ \\
& TBI slow & 7 & $0.764(.065)$ & $1.731(0.405)$ & $2.169(0.406)$ & $2.846(0.649)$
\end{tabular}

*Pair-wise statistically significant difference, $p<0.05$.

fMRI, functional magnetic resonance imaging; WML, working memory load; SD, standard deviation; TBI, traumatic brain injury; IHTT, interhemispheric transfer time. 
statistically significant difference between the TBI groups for injury severity, nor time post-injury.

\section{Behavioral functional magnetic resonance imaging data}

Means, SDs, and pair-wise comparisons for accuracy and response speed are presented in Table 2, and results are also plotted in Figure 1 . There were no statistically significant $\mathrm{WML} \times$ group interactions for any of the behavioral measures. There were, however, statistically significant main effects of WML, revealing a linear decrease in performance as measured by response speed and accuracy with higher WML. The only statistically significant effect of group was found for response speed at WML 3 and 4, where the TBI normal group had slower response times than controls.

\section{Blood oxygen level dependent functional magnetic resonance imaging data}

The TBI slow group exhibited BOLD hyperactivation as compared to both the TBI normal group and controls in widespread brain areas encompassing frontal, parietal, and occipital regions (Table 3; Fig. 2). There were no regions where the TBI normal group or controls had statistically significantly higher BOLD activation than the TBI slow group. The TBI normal group exhibited BOLD hypoactivation compared to controls, predominantly in posterior brain regions (Table 3; Fig. 2). There were no regions where the TBI normal group had statistically significantly higher BOLD activation than controls.

In the full TBI sample, slower IHTT (treated as a continuous variable) was associated with increased BOLD activation in widespread brain regions (Table 4; Fig. 3). Less severe injury (as measured by GCS at admission) was also associated with increased BOLD activation (Table 4; Fig. 3). The associations between BOLD activation and age, time post-injury, or the clinical neurocognitive performance index in the TBI group were not statistically significant. In controls, older age was associated with increased BOLD activation in predominantly right posterior brain regions (Table 4; Fig. 4). Also, in controls, better neurocognitive function as measured by the CPI was associated with increased BOLD activation in left temporo-occipital regions (Table 4; Fig. 4). Slower IHTT in controls was associated with increased BOLD activation bilaterally in occipitoparietal regions (Table 4; Fig. 4).

\section{Discussion}

This study demonstrated that BOLD hyperactivation in children with msTBI is associated with an electrophysiological ERP measure of IHTT. A subgroup of msTBI children with slow IHTT had greater BOLD activation with higher WML than both healthy control children and a subgroup of msTBI children with normal IHTT. The same effect was also demonstrated in the full TBI sample, where slower IHTT modeled as a continuous variable was associated with widespread BOLD hyperactivations. BOLD hyperactivations are commonly observed after msTBI, and here we provide the first compelling evidence on how such activations are linked to a more direct measure of neuronal function.

Increased BOLD activations are hypothesized to represent increased metabolic demands in the brain. These can arise from increased task load (e.g., WML), but also from suboptimal neuronal transfer of information, ${ }^{1,2}$ which in our study was operationalized as reduced IHTT. Our main contrast of interest was the linear increase in activation with higher WML in a parametric fMRI design. Behavioral results confirmed the expected WML-dependent linear decrease in accuracy and increase in response latency. In the fMRI analyses, all groups also demonstrated the expected effect, with more pronounced BOLD activations with greater WML.

BOLD hyperactivation in TBI individuals with slower IHTT may indicate that they had to recruit more neuronal resources to maintain task performance comparable to that of controls, as the working memory load increased. This likely reflects the presence of compensatory mechanisms; processes that in this study may have mitigated the effect of brain injury on cognitive performance during fMRI. A link between slower IHTT and increased BOLD activation
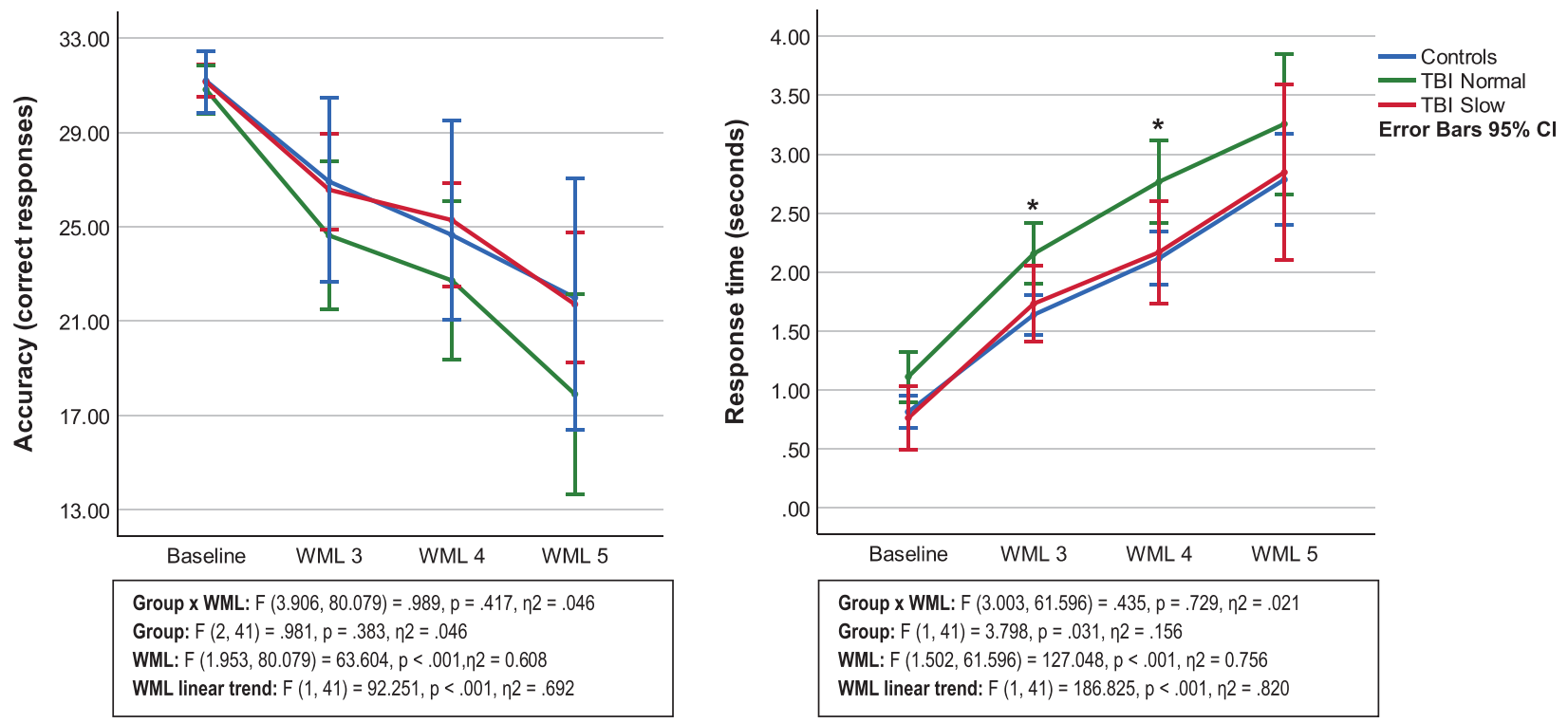

Group x WML: $F(3.003,61.596)=.435, p=.729, \eta 2=.021$

Group: $F(1,41)=3.798, p=.031, \eta 2=.156$

WML: $F(1.502,61.596)=127.048, p<.001, \eta 2=0.756$

WML linear trend: $F(1,41)=186.825, p<.001, \eta 2=.820$

FIG. 1. Working memory fMRI task performance. *Pair-wise statistically significant difference between the TBI normal group and controls, $p<0.05$. CI, confidence interval; fMRI, functional magnetic resonance imaging; TBI, traumatic brain injury; WML, working memory load. 
Table 3. Between-Group Differences in BOLD Activation

\begin{tabular}{|c|c|c|c|c|c|c|}
\hline \multirow[b]{2}{*}{ Anatomical region } & \multirow[b]{2}{*}{$R / L$} & \multirow[b]{2}{*}{ Size in number of voxels } & \multirow[b]{2}{*}{$Z$} & \multicolumn{3}{|c|}{ Coordinates for peak activation (MNI) } \\
\hline & & & & $X$ & $Y$ & $Z$ \\
\hline \multicolumn{7}{|l|}{ TBI slow > control } \\
\hline Frontal pole & $\mathrm{R}$ & 6603 & 6.01 & 34 & 38 & 30 \\
\hline Frontal pole & $\mathrm{R}$ & $\operatorname{lm}$ & 5.75 & 32 & 38 & 34 \\
\hline Supramarginal gryus, anterior division & $\mathrm{R}$ & $\operatorname{lm}$ & 5.48 & 58 & -24 & 50 \\
\hline Inferior frontal gyrus, pars triangularis & $\mathrm{R}$ & $\operatorname{lm}$ & 5.46 & 58 & 26 & -4 \\
\hline Frontal pole & $\mathrm{R}$ & $\operatorname{lm}$ & 5.40 & 36 & 38 & 34 \\
\hline Middle frontal gyrus & $\mathrm{R}$ & $\operatorname{lm}$ & 5.39 & 32 & 34 & 36 \\
\hline Lateral occipital cortex, superior division & $\mathrm{L}$ & 1644 & 5.67 & -18 & -70 & 58 \\
\hline Lateral occipital cortex, superior division & $\mathrm{L}$ & $\operatorname{lm}$ & 5.11 & -16 & -78 & 50 \\
\hline Superior parietal lobule & $\mathrm{L}$ & $\operatorname{lm}$ & 4.76 & -22 & -46 & 58 \\
\hline Superior parietal lobule & $\mathrm{L}$ & $\operatorname{lm}$ & 4.65 & -22 & -48 & 62 \\
\hline Superior parietal lobule & $\mathrm{L}$ & $\operatorname{lm}$ & 4.55 & -28 & -46 & 58 \\
\hline Lateral occipital cortex, superior division & $\mathrm{L}$ & $\operatorname{lm}$ & 4.50 & -10 & -66 & 60 \\
\hline Frontal pole & $\mathrm{L}$ & 978 & 4.66 & -36 & 46 & 32 \\
\hline Insular cortex & $\mathrm{L}$ & $\operatorname{lm}$ & 4.43 & -30 & 18 & 6 \\
\hline Middle frontal gyrus & $\mathrm{L}$ & $\operatorname{lm}$ & 4.29 & -42 & 38 & 30 \\
\hline Middle frontal gyrus & $\mathrm{L}$ & $\operatorname{lm}$ & 4.09 & -32 & 34 & 36 \\
\hline Middle frontal gyrus & $\mathrm{L}$ & $\operatorname{lm}$ & 4.05 & -30 & 24 & 36 \\
\hline Frontal pole & $\mathrm{L}$ & $\operatorname{lm}$ & 3.83 & -40 & 44 & 20 \\
\hline Superior frontal gyrus & $\mathrm{R}$ & 930 & 4.37 & 20 & 2 & 52 \\
\hline Cingulate gyrus, anterior division & $\mathrm{L}$ & $\operatorname{lm}$ & 3.89 & -4 & 8 & 40 \\
\hline Supplementary motor cortex & $\mathrm{R}$ & $\operatorname{lm}$ & 3.83 & 6 & -8 & 54 \\
\hline Supplementary motor cortex & $\mathrm{L}$ & $\operatorname{lm}$ & 3.69 & -10 & 0 & 50 \\
\hline Supplementary motor cortex & $\mathrm{R} / \mathrm{L}$ & $\operatorname{lm}$ & 3.68 & 0 & 4 & 52 \\
\hline Supplementary motor cortex & $\mathrm{R}$ & $\operatorname{lm}$ & 3.62 & 6 & -2 & 54 \\
\hline \multicolumn{7}{|l|}{ TBI slow > TBI normal } \\
\hline Lateral occipital cortex, superior division & $\mathrm{L}$ & 17,424 & 6.50 & -22 & -68 & 58 \\
\hline Lateral occipital cortex, superior division & $\mathrm{L}$ & $\operatorname{lm}$ & 5.81 & -18 & -70 & 60 \\
\hline Lateral occipital cortex, superior division & $\mathrm{L}$ & $\operatorname{lm}$ & 5.49 & -12 & -68 & 60 \\
\hline Precuneus cortex & $\mathrm{L}$ & $\operatorname{lm}$ & 5.35 & -6 & -68 & 54 \\
\hline Postcentral gyrus & $\mathrm{R}$ & $\operatorname{lm}$ & 5.30 & 12 & -46 & 72 \\
\hline Superior parietal lobule & $\mathrm{L}$ & $\operatorname{lm}$ & 5.27 & -22 & -46 & 58 \\
\hline Frontal orbital cortex & $\mathrm{L}$ & 5891 & 5.63 & -24 & 18 & -10 \\
\hline Precentral gyrus & $\mathrm{L}$ & $\operatorname{lm}$ & 4.93 & -56 & 4 & 4 \\
\hline Middle frontal gyrus & $\mathrm{L}$ & $\operatorname{lm}$ & 4.59 & -36 & 24 & 26 \\
\hline Insular cortex & $\mathrm{L}$ & $\operatorname{lm}$ & 4.49 & -30 & 16 & 2 \\
\hline Precentral gyrus & $\mathrm{L}$ & $\operatorname{lm}$ & 4.47 & -54 & 4 & 34 \\
\hline Insular cortex & $\mathrm{L}$ & $\operatorname{lm}$ & 4.46 & -32 & 16 & 6 \\
\hline Lateral occipital cortex, superior division & $\mathrm{L}$ & 3513 & 4.58 & -28 & -76 & 22 \\
\hline Intracalcarine cortex & $\mathrm{L}$ & $\operatorname{lm}$ & 4.42 & -6 & -86 & 6 \\
\hline Lateral occipital cortex, superior division & $\mathrm{L}$ & $\operatorname{lm}$ & 4.12 & -32 & -78 & 18 \\
\hline Occipital pole & $\mathrm{L}$ & $\operatorname{lm}$ & 3.99 & -8 & -96 & 4 \\
\hline Intracalcarine cortex & $\mathrm{R}$ & $\operatorname{lm}$ & 3.97 & 14 & -80 & 6 \\
\hline Cerebellum (left VI) & $\mathrm{L}$ & $\operatorname{lm}$ & 3.93 & -24 & -52 & -34 \\
\hline \multicolumn{7}{|l|}{ Controls > TBI normal } \\
\hline Occipital fusiform gyrus & $\mathrm{R}$ & 4709 & 5.09 & 22 & -76 & -22 \\
\hline Cuneal cortex & $\mathrm{R}$ & $\operatorname{lm}$ & 4.72 & 10 & -80 & 32 \\
\hline Cuneal cortex & $\mathrm{R}$ & $\operatorname{lm}$ & 4.68 & 10 & -76 & 32 \\
\hline Occipital fusiform gyrus & $\mathrm{R}$ & $\operatorname{lm}$ & 4.47 & 26 & -76 & -18 \\
\hline Lateral occipital cortex, superior division & $\mathrm{R}$ & $\operatorname{lm}$ & 4.45 & 20 & -62 & 54 \\
\hline Lateral occipital cortex, superior division & $\mathrm{L}$ & $\operatorname{lm}$ & 2.26 & -30 & -66 & 20 \\
\hline Postcentral gyrus & $\mathrm{L}$ & 1285 & 4.13 & -62 & -22 & 32 \\
\hline Parietal operculum cortex & $\mathrm{L}$ & $\operatorname{lm}$ & 3.99 & -50 & -26 & 14 \\
\hline Planum temporale & $\mathrm{L}$ & $\operatorname{lm}$ & 3.95 & -44 & -34 & 14 \\
\hline Supramarginal gyrus, anterior division & $\mathrm{L}$ & $\operatorname{lm}$ & 3.88 & -46 & -36 & 42 \\
\hline Superior parietal lobule & $\mathrm{L}$ & $\operatorname{lm}$ & 3.83 & -36 & -42 & 64 \\
\hline Supramarginal gyrus, anterior division & $\mathrm{L}$ & $\operatorname{lm}$ & 3.81 & -50 & -38 & 42 \\
\hline
\end{tabular}


TABle 3. (Continued)

\begin{tabular}{llccccc}
\hline & & & & \multicolumn{3}{c}{ Coordinates for peak activation (MNI) } \\
\cline { 6 - 7 } Anatomical region & $R / L$ & Size in number of voxels & $Z$ & $X$ & $Y$ & $Z$ \\
\hline Superior parietal lobule & $\mathrm{L}$ & 949 & 4.52 & -28 & -54 & 48 \\
$\quad$ Lateral occipital cortex, superior division & $\mathrm{L}$ & $\mathrm{m}$ & 4.00 & -28 & -58 & 60 \\
Superior parietal lobule & $\mathrm{L}$ & $\mathrm{lm}$ & 3.90 & -20 & -52 & 50 \\
Superior parietal lobule & $\mathrm{L}$ & $\mathrm{lm}$ & 3.88 & -30 & -46 & 44 \\
Superior parietal lobule & $\mathrm{L}$ & $1 \mathrm{~m}$ & 3.84 & -24 & -56 & 50 \\
$\quad$ Superior parietal lobule & $\mathrm{L}$ & $\mathrm{lm}$ & 3.81 & -30 & -54 & 56 \\
Controls > TBI slow & - & - & - & - & - & - \\
TBI normal > controls & - & - & - & - & - & - \\
TBI normal > TBI slow & - & - & - & - & - & - \\
\hline
\end{tabular}

Results were achieved using a mixed-effects model corrected for multiple comparisons using a cluster threshold of $\mathrm{Z}>2.3$ and a corrected cluster significance threshold of $p<0.05$. Main peaks and up to five local maxima $(\mathrm{lm})$ within each cluster are reported. Naming of anatomical regions was based on the Harvard Oxford cortical and subcortical structural atlases as implemented in the FSL software. Note that some clusters are relatively large and therefore span over several brain regions (see Fig. 1 for more details).

BOLD, blood oxygen level dependent; TBI traumatic brain injury; MNI, Montreal Neurological Institute.

was also confirmed within the healthy control group. Importantly, there were no group $\times$ WML interaction effects for online fMRI task performance, given that this could potentially compromise the interpretation of BOLD group comparisons. ${ }^{19}$ To further minimize any effects of more subtle performance differences, we only included correct trials in our analyses. Given that there were no demographic or clinical differences between the slow and normal IHTT groups, the BOLD hyperactivations in the msTBI children with slow IHTT is not likely to be explained by poor effort, demographics, or injury-related differences.

Neurovascular coupling can be affected after brain injury, potentially even in the chronic phase, ${ }^{4,7}$ which has provided some uncertainty about the interpretation of the functional role of BOLD alterations after msTBI. Here, we took an alternative approach to determining the role of BOLD alterations in msTBI by evaluating their link to IHTT-a basic electrophysiological measure of neuronal function. No previous studies have investigated associations between BOLD fMRI and electrophysiological data in msTBI. In a study combining data from adults with mild TBI and matched healthy controls, decreased amplitude in the event-related N350 component derived from a working memory task was associated with smaller BOLD signal changes in a corresponding fMRI task. ${ }^{1}$ However, this association could not be reproduced separately in the mild TBI group, leaving it an open question whether this association was linked to the brain injury or not. Longer IHTT is associated with slower transfer of visual information across posterior visual brain regions, particularly relying on pathways in the posterior corpus callosum. ${ }^{25}$ Higher-order cognitive processes such as working memory - and in particular those that are computationally demanding (e.g., higher WML) — rely on coordinated processing nodes in both hemispheres. ${ }^{18}$ A possible explanation of our findings is that impaired IHTT after msTBI may reflect disrupted long-range interhemispheric collaboration, which, in turn, causes increased reliance on local or core networks. Support for this has been found in studies observing decreased global connectivity in the context of increased local connectivity after msTBI. ${ }^{26,27}$ Moreover, in adult TBI, hyperconnectivity seems to occur in what is typically observed to be the most highly connected core regions in the brain, also referred to as the "rich club." 28 The rich club is a high-cost, high-capacity feature of brain connectivity central for global brain communication that encompass brain regions such as the anteri- or/posterior cingulate cortex, superior frontal cortex, the insula, and precuneus, ${ }^{29}$ some of which were implicated in the current study.

Interestingly, our preliminary analyses of structural connectivity in an overlapping sample show that slow IHTT is, in fact, linked to rich club hyperconnectivity at the expense of reduced peripheral integrity. ${ }^{30}$ An alternative, but non-mutually exclusive, mechanism is that the BOLD alterations observed in the current study are caused by a more global effect of white matter injury, beyond the corpus callosum. Reduced corpus callosum integrity after msTBI is typically indicative of more widespread white matter disorganization attributable to traumatic axonal injury caused by acceleration/deceleration and rotational forces. ${ }^{31}$

Despite examples of studies in pediatric msTBI examining relevant cognitive constructs (e.g., working memory) through the design of the fMRI task, few have investigated links to external clinical measures of neurocognitive function. Previous studies including individuals with pediatric msTBI have indicated both positive and negative relationships between BOLD activations and external measures of neurocognitive function. ${ }^{2,3}$ However, these results cannot be directly attributed to msTBI, given that orthopedic controls $^{2}$ or patients with other types of acquired brain injuries ${ }^{3}$ were also included in the analyses. Cognitive dysfunction is common after pediatric msTBI, ${ }^{20,33}$ and was also confirmed in our subsample, because both TBI groups performed worse than controls on a clinical CPI. In controls, better performance on the CPI was associated with more pronounced BOLD activation in temporo-occipital regions. However, we failed to confirm a direct link between BOLD activation and the clinical neurocognitive performance index for the TBI group as a whole.

Brain reorganization and compensatory mechanisms are expected to develop over time as spontaneous recovery and experience-dependent new learning occurs. ${ }^{1,34}$ Relatively large variability in time post-injury for the subset of patients included in the fMRI analyses could therefore potentially have affected the BOLD-function results. This is unlikely, however, given that we found no significant association between time post-injury and BOLD activation. Differences in task demands between the fMRI task and the CPI (which is a broader measure of cognitive function) may also have obscured the BOLD-function relationship, given that compensatory mechanisms after msTBI may be more relevant for some tasks than others. Performance was highly similar across all 
TBI Slow $(n=7)>$ Controls $(n=26)$
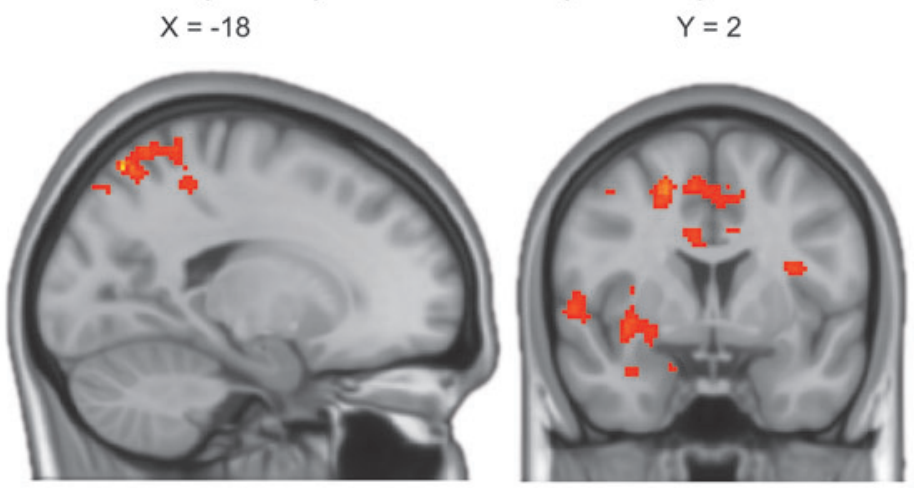

Right - Left

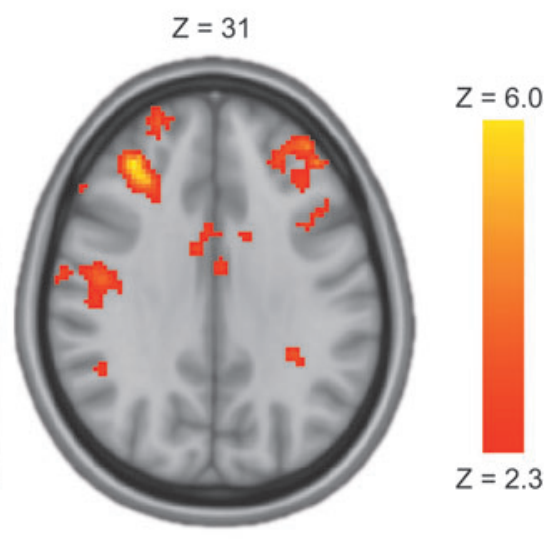

TBI Slow $(n=7)>$ TBI Normal $(n=11)$
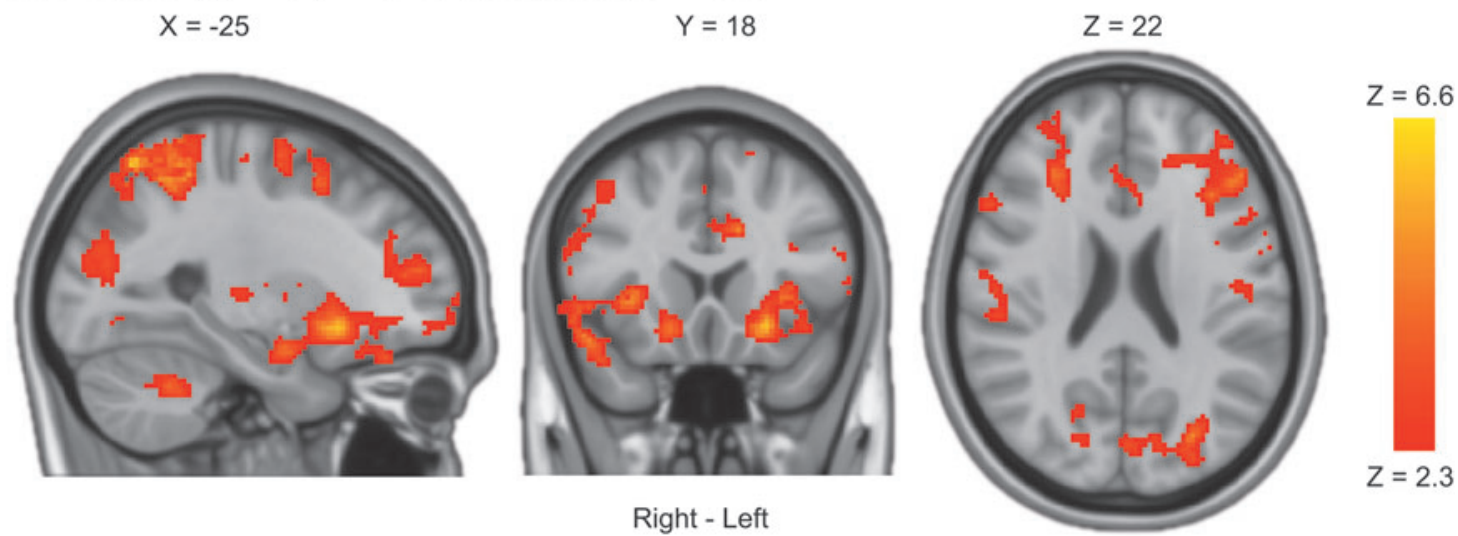

Right - Left

Controls $(n=26)>$ TBI Normal $(n=11)$
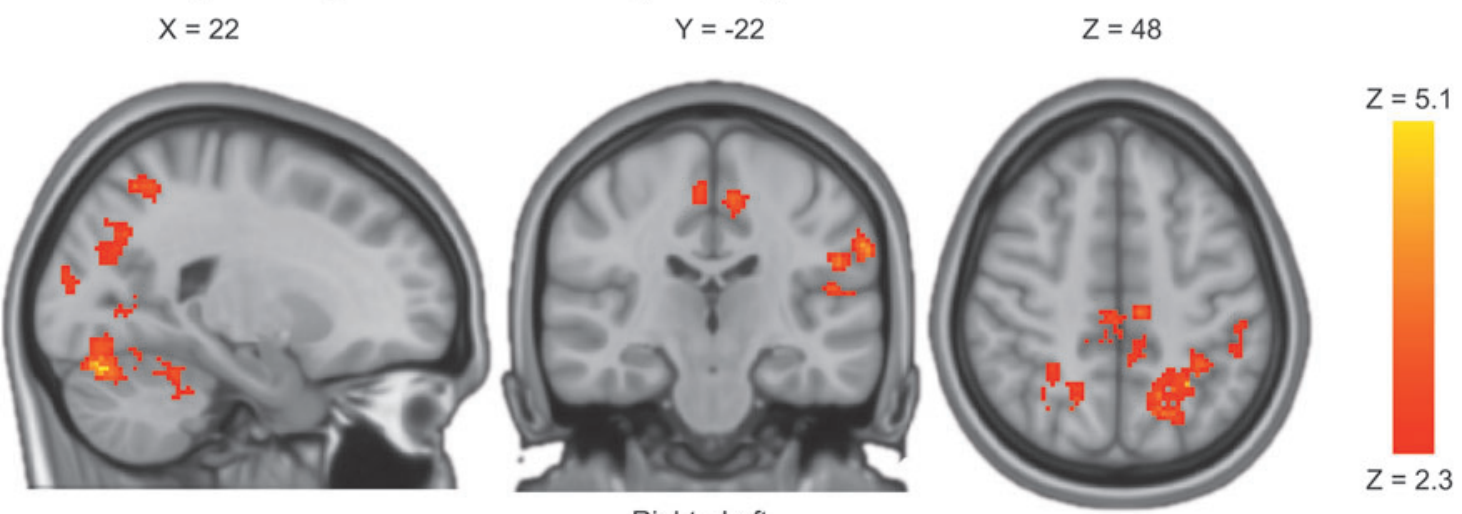

Right - Left

FIG. 2. Between-group differences in BOLD activation. Statistical parametric maps were achieved using a mixed-effects model corrected for multiple comparisons using a cluster threshold of $\mathrm{Z}>2.3$ and a corrected cluster significance threshold of $p<0.05$. Only statistically significant results are shown. There were no regions where the TBI normal group or controls had statistically significant higher BOLD activation than the TBI slow group. There were no regions where the TBI normal group had statistically significant higher BOLD activation than controls. BOLD, blood oxygen level dependent; TBI, traumatic brain injury.

groups in the spatial working memory task, and we only included correct responses in the fMRI analyses. Although important for the interpretation of BOLD activations, ${ }^{19}$ similar task performance may also indicate that the task difficulty was lower in the fMRI task than for the tasks combined in the CPI.

TBI children with slow and normal IHTT did not differ on the CPI, but both groups performed more poorly than healthy controls. Because the two msTBI groups had similar performance (and did not differ on any other clinical and demographic variables), but were separable based on their levels of activation, we extended our analyses to include ad hoc investigation of within-group associations between BOLD activations and the CPI (Fig. 5). Better cognitive performance as measured by the CPI was associated with pronounced widespread BOLD activation in the slow IHTT msTBI children. In msTBI children with normal IHTT, this relationship was limited to activation in a small region in the post-central gyrus. 
Coordinates for peak activation (MNI)

\begin{tabular}{|c|c|c|c|c|c|c|}
\hline \multirow{2}{*}{ Anatomical region } & \multirow[b]{2}{*}{$R / L$} & \multirow[b]{2}{*}{ Size in number of voxels } & \multirow[b]{2}{*}{$Z$} & \multicolumn{3}{|c|}{ 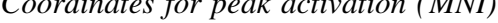 } \\
\hline & & & & $X$ & $Y$ & $Z$ \\
\hline \multicolumn{7}{|l|}{ TBI $(n=18)$} \\
\hline Age & - & - & - & - & - & - \\
\hline Cognitive performance index (CPI) & - & - & - & - & - & - \\
\hline \multicolumn{7}{|l|}{ Interhemispheric transfer time (IHTT) } \\
\hline Supplementary motor cortex & $\mathrm{L}$ & 13,621 & 5.74 & -2 & 4 & 50 \\
\hline Superior parietal lobule & $\mathrm{L}$ & & 5.05 & -26 & -52 & 52 \\
\hline Superior parietal lobule & $\mathrm{R}$ & & 4.95 & 40 & -44 & 54 \\
\hline Supramarginal gyrus, posterior division & $\mathrm{R}$ & & 4.92 & 46 & -44 & 58 \\
\hline Supplementary motor cortex & $\mathrm{L}$ & & 4.87 & -2 & 0 & 56 \\
\hline Lateral occipital cortex, superior division & $\mathrm{L}$ & & 4.84 & -16 & -70 & 58 \\
\hline Frontal pole & $\mathrm{L}$ & 1673 & 4.42 & -26 & 48 & 10 \\
\hline Middle frontal gyrus & $\mathrm{L}$ & & 4.11 & -36 & 30 & 30 \\
\hline Middle frontal gyrus & $\mathrm{L}$ & & 4.11 & -34 & 24 & 26 \\
\hline Middle frontal gyrus & $\mathrm{L}$ & & 3.67 & -40 & 34 & 28 \\
\hline Middle frontal gyrus & $\mathrm{L}$ & & 3.66 & -30 & 34 & 38 \\
\hline Frontal orbital cortex & $\mathrm{L}$ & & 3.63 & -24 & 18 & -10 \\
\hline \multicolumn{7}{|l|}{ Injury severity (GCS at admission) } \\
\hline Precentral gyrus & $\mathrm{L}$ & 12,077 & 5.42 & -54 & -4 & 46 \\
\hline Middle frontal gyrus & $\mathrm{L}$ & & 5.29 & -50 & 28 & 38 \\
\hline Frontal pole & $\mathrm{R}$ & & 5.07 & 28 & 36 & 28 \\
\hline Frontal pole & $\mathrm{L}$ & & 5.04 & -32 & 52 & 18 \\
\hline Precentral gyrus & $\mathrm{L}$ & & 5.01 & -52 & 6 & 40 \\
\hline Middle frontal gyrus & $\mathrm{R}$ & 1933 & 5.37 & 50 & 8 & 42 \\
\hline Precentral gyrus & $\mathrm{R}$ & & 5.26 & 58 & 6 & 38 \\
\hline Postcentral gyrus & $\mathrm{R}$ & & 4.58 & 64 & -14 & 40 \\
\hline Middle frontal gyrus & $\mathrm{R}$ & & 4.40 & 46 & 8 & 48 \\
\hline Supramarginal gyrus, posterior division & $\mathrm{R}$ & & 4.06 & 52 & -36 & 56 \\
\hline Postcentral gyrus & $\mathrm{R}$ & & 3.93 & 60 & -16 & 44 \\
\hline Time post-injury & - & - & - & - & - & - \\
\hline \multicolumn{7}{|l|}{ Controls $(n=26)$} \\
\hline \multicolumn{7}{|l|}{ Age } \\
\hline Superior parietal lobule & $\mathrm{R}$ & 1288 & 4.49 & 44 & -38 & 60 \\
\hline Postcentral gyrus & $\mathrm{R}$ & & 3.94 & 44 & -36 & 54 \\
\hline Postcentral gyrus & $\mathrm{R}$ & & 3.92 & 52 & -28 & 52 \\
\hline Precentral gyrus & $\mathrm{R}$ & & 3.73 & 32 & -18 & 68 \\
\hline Superior parietal lobule & $\mathrm{R}$ & & 3.70 & 40 & -40 & 56 \\
\hline Postcentral gyrus & $\mathrm{R}$ & & 3.62 & 42 & -32 & 52 \\
\hline \multicolumn{7}{|l|}{ IHTT } \\
\hline Lateral occipital cortex, superior division & $\mathrm{L}$ & & 4.66 & -8 & -80 & 46 \\
\hline Precuneous cortex & $\mathrm{R}$ & & 4.53 & 6 & -74 & 52 \\
\hline Lateral occipital cortex, superior division & $\mathrm{R}$ & & 4.48 & 14 & -80 & 52 \\
\hline Lateral occipital cortex, superior division & $\mathrm{R}$ & & 4.39 & 18 & -80 & 50 \\
\hline Precuneous cortex & $\mathrm{L}$ & & 4.25 & -8 & -76 & 46 \\
\hline Precuneous cortex & $\mathrm{L}$ & & 4.16 & -6 & -80 & 52 \\
\hline \multicolumn{7}{|l|}{ CPI } \\
\hline Occipital fusiform gyrus & $\mathrm{L}$ & 869 & 3.68 & -26 & -90 & -12 \\
\hline Occipital pole & $\mathrm{L}$ & & 3.58 & -22 & -94 & -14 \\
\hline Occipital fusiform gyrus & $\mathrm{L}$ & & 3.53 & -24 & -90 & -18 \\
\hline Inferior temporal gyrus, temporooccipital part & $\mathrm{L}$ & & 3.48 & -52 & -54 & -28 \\
\hline Temporal occipital fusiform cortex & $\mathrm{L}$ & & 3.48 & -36 & -56 & -22 \\
\hline Cerebellum, left crus I & $\mathrm{L}$ & & 3.48 & -28 & -84 & -32 \\
\hline Superior temporal gyrus, posterior division & $\mathrm{L}$ & 728 & 4.52 & -54 & -40 & 6 \\
\hline Supramarginal gyrus, posterior division & $\mathrm{L}$ & & 3.88 & -48 & -46 & 22 \\
\hline Planum temporale & $\mathrm{L}$ & & 3.69 & -42 & -40 & 18 \\
\hline Middle temporal gyrus, posterior division & $\mathrm{L}$ & & 3.56 & -60 & -38 & -6 \\
\hline Middle temporal gyrus, posterior division & $\mathrm{L}$ & & 3.55 & -56 & -40 & -6 \\
\hline Parietal operculum cortex & $\mathrm{L}$ & & 3.54 & -44 & -44 & 24 \\
\hline
\end{tabular}

Results were achieved using a mixed-effects model corrected for multiple comparisons using a cluster threshold of $\mathrm{Z}>2.3$ and a corrected cluster significance threshold of $p<0.05$. Main peaks and up to five local maxima $(\mathrm{lm})$ within each cluster are reported. Naming of anatomical regions was based on the Harvard Oxford cortical and subcortical structural atlases as implemented in the FSL software. Note that some clusters are relatively large and therefore span over several brain regions (see Figs. 3 and 4 for more details).

TBI traumatic brain injury; GCS, Glasgow Coma Scale; MNI, Montreal Neurological Institute. 
Positive BOLD-function associations therefore seem stronger within the slow-IHTT children, which provides indirect evidence that hyperactivations observed in the between-group analyses may be compensatory.

The observed BOLD hyperactivations in msTBI children with slow IHTT could not be explained by having sustained a brain injury by itself, nor by injury severity (i.e., GCS). More severe injury as measured by GCS score in the whole TBI sample was associated with BOLD hypoactivations, and TBI patients with normal IHTT exhibited lower WML-dependent BOLD activation compared to healthy controls. TBI children with normal IHTT had statistically significant slower response times at WML 3 and 4 during the fMRI task than healthy controls. Moreover, although not statistically significant, the TBI normal group also showed a trend of slower response times compared to the TBI slow group. This is somewhat counterintuitive, given that one would expect those with slow IHTT to have the slowest response times, especially given that faster IHTT is considered a proxy for better white matter integrity/organization, which may facilitate processing speed.

However, interpretation of differences in response time in the context of similar accuracy on a spatial working memory task is not straightforward. Increased response time also means that the subject must maintain information in their working memory longer (i.e., increased task difficulty) than if they are able to respond faster. Interestingly, earlier research has shown that increased BOLD activation after adult msTBI can be partially attributed to a transient normal response that is also observed in healthy participants attributable to slowed information processing. ${ }^{6}$ BOLD hyperactivations have therefore been suggested to be partly explained by increased on-task "cycle-time," which is typically reflected in increased behavioral response time. ${ }^{6,7}$ If this was the case in our study, the TBI normal group would be expected to show hyperactivations rather than hypoactivations. It is therefore unlikely that the seemingly compensatory hyperactivations in the slow TBI group are driven by subtle behavioral differences in response time between the groups. However, given that the electrophysiological IHTT measure is a more direct reflection of the basic processing capacity in the brain, BOLD hyperactivation as observed in the TBI slow group may still reflect increased on-task "neuronal cycle time," possibly representing recruitment of latent support mechanisms that are activated given that the task is more slowly processed on a neuronal level. ${ }^{6,7}$

\section{Within-group associations TBI $(\mathrm{n}=18)$}

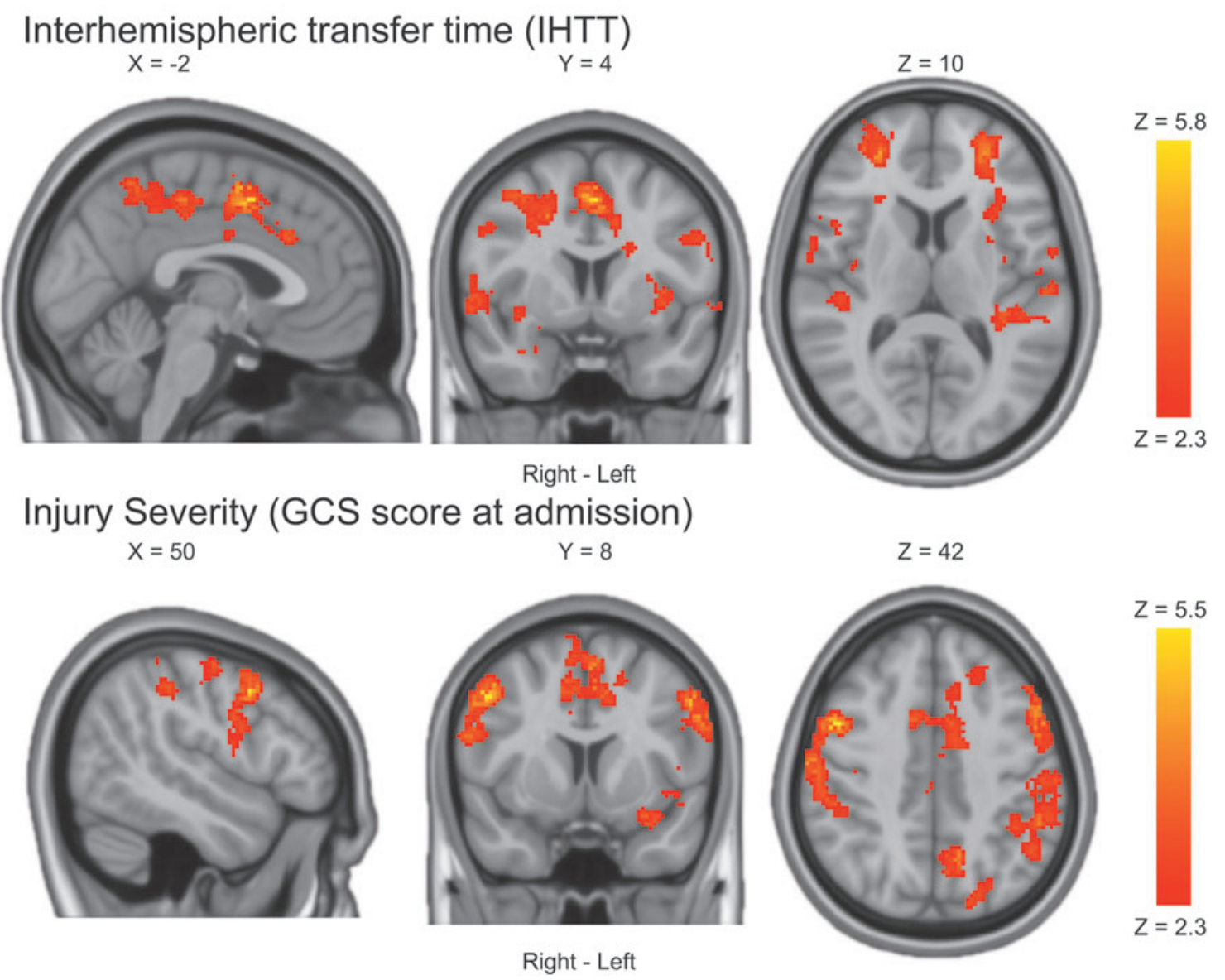

FIG. 3. Within-group associations in the full TBI sample. Statistical parametric maps were achieved using a mixed-effects model corrected for multiple comparisons using a cluster threshold of $\mathrm{Z}>2.3$ and a corrected cluster significance threshold of $p<0.05$. Only statistically significant results are shown. There was no statistically significant association between BOLD activation and age, time postinjury, nor the clinical neurocognitive performance index in the TBI group. BOLD, blood oxygen level dependent; GCS, Glasgow Coma Scale; IHTT, interhemispheric transfer time; TBI, traumatic brain injury. 


\section{Within-group associations Controls $(n=26)$}
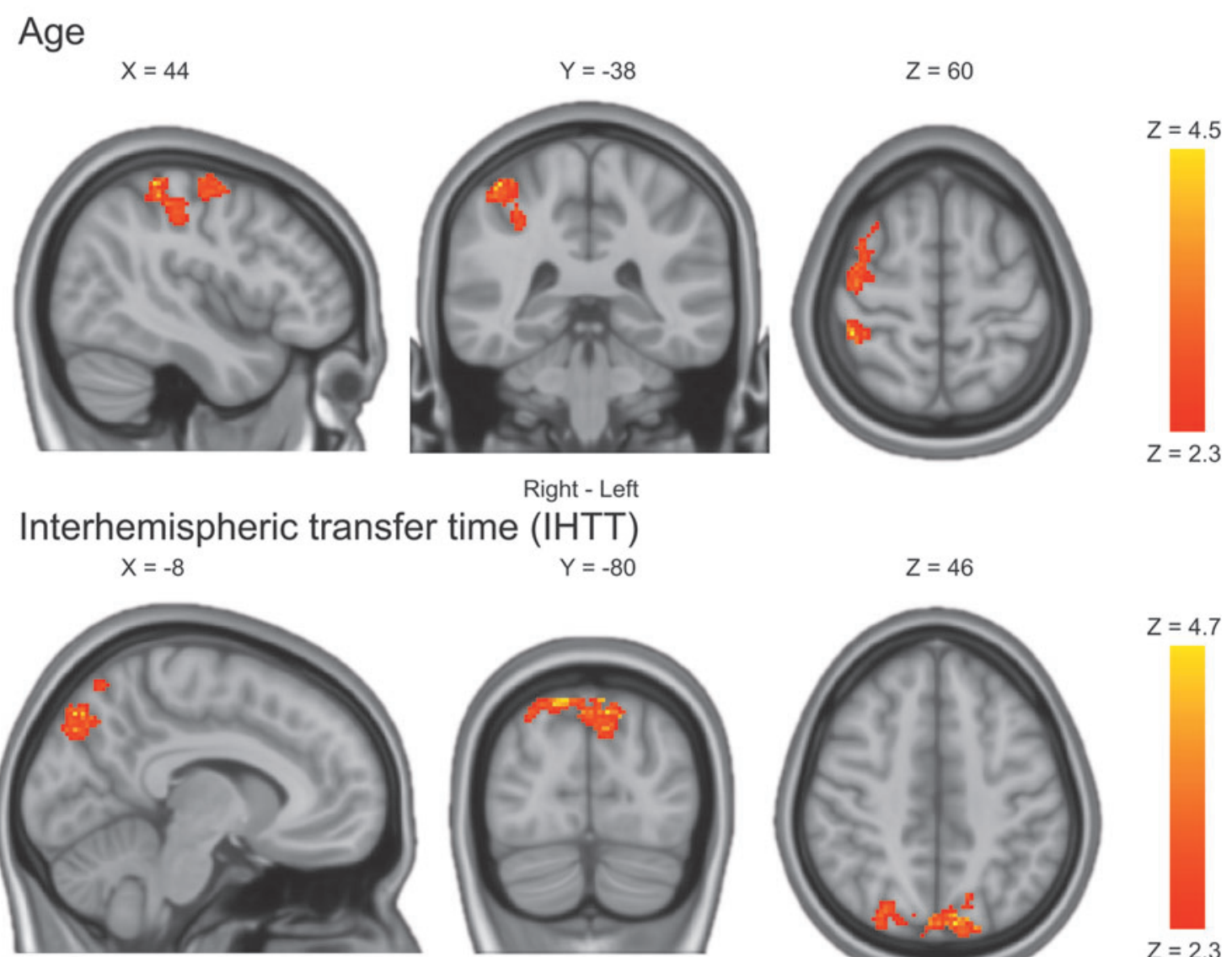

Right - Left

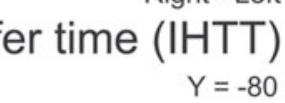

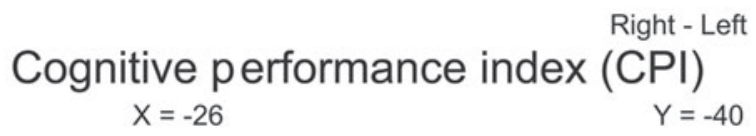

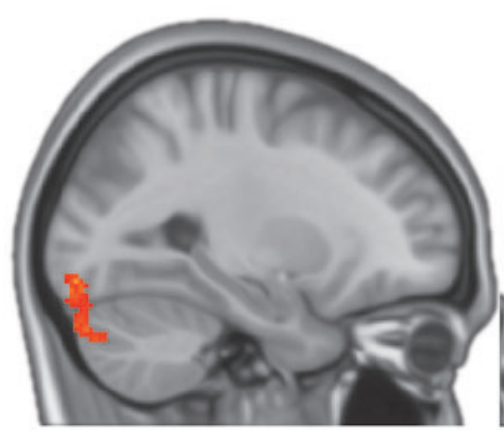

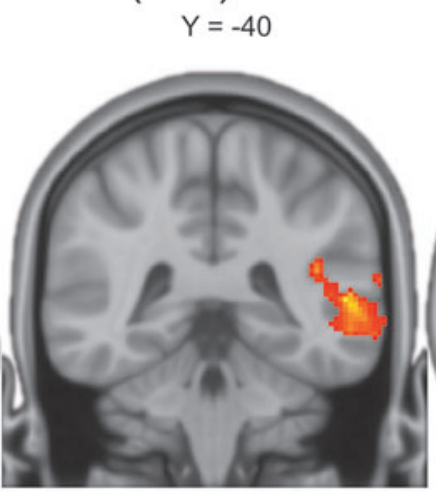

Right - Left
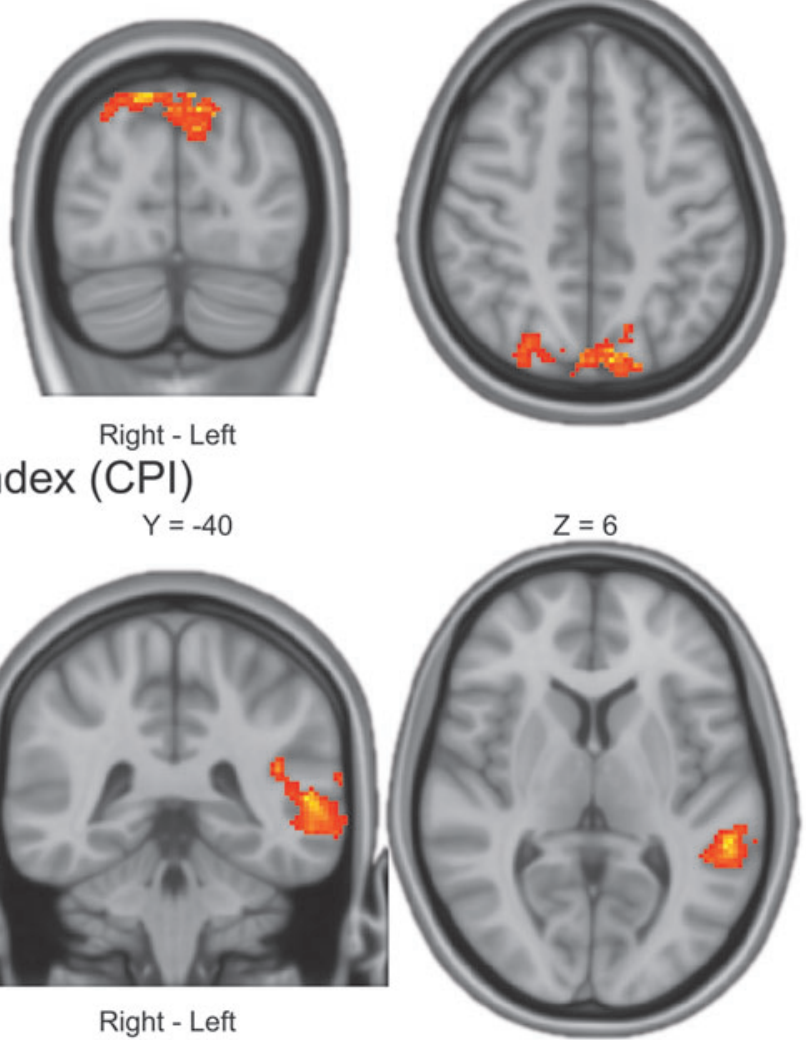

$Z=46$

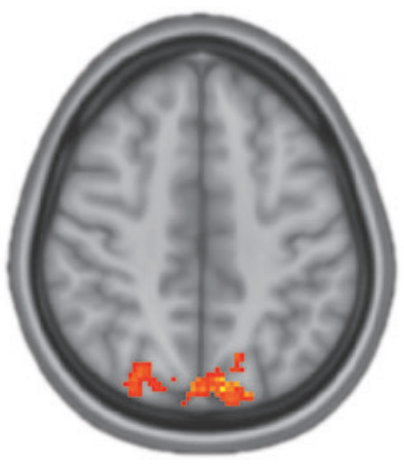

$Z=4.7$

$Z=4.6$

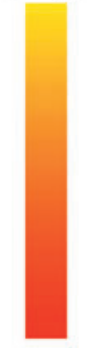

$Z=2.3$

FIG. 4. Within-group associations in controls. Statistical parametric maps were achieved using a mixed-effects model corrected for multiple comparisons using a cluster threshold of $\mathrm{Z}>2.3$ and a corrected cluster significance threshold of $p<0.05$. Only statistically significant results are shown.

Our results provide important new directions to interpreting BOLD alterations after pediatric msTBI, but also reflect the highly heterogeneous nature of msTBI, and implies the existence of several potentially non-mutually exclusive mechanisms affecting the BOLD signal (e.g., injury severity, compensatory neuronal processes, or inefficient neuronal processing). Despite including a larger total sample size than most previous fMRI studies of pediatric msTBI, subgroup analysis in our study is still limited by a modest sample size. Our primary aim was to investigate hypotheses regarding the previously identified slow IHTT subgroup, but the statistical power to evaluate more subtle multivariate and possibly non-linear relationships was limited. It is also possible that some of the null findings in our study are type 2 errors, as in similarly powered fMRI studies. ${ }^{35}$

BOLD fMRI studies of pediatric msTBI have now been conducted for just over a decade, ${ }^{6}$ but are still few, and have included 


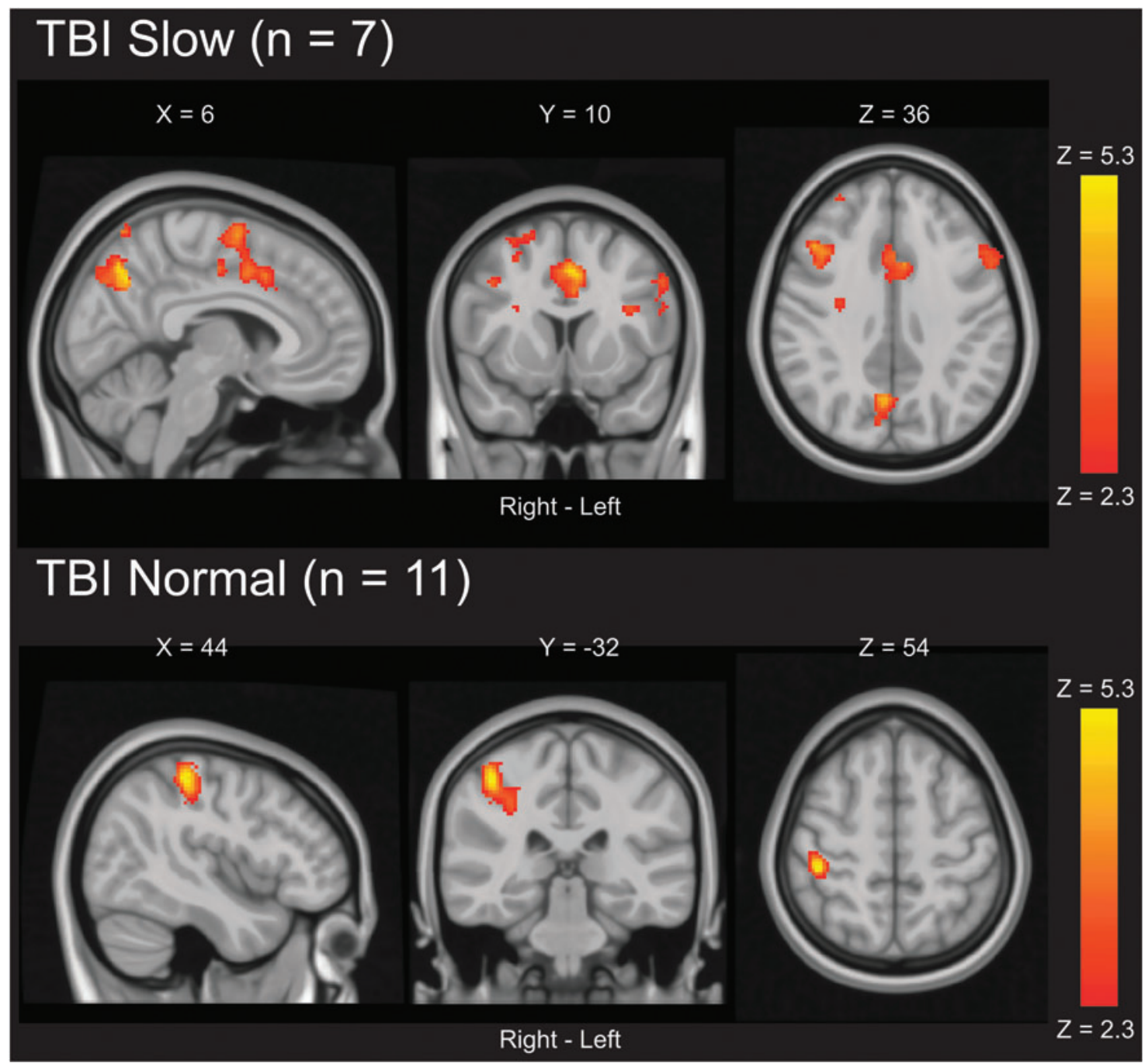

FIG. 5. Ad-hoc within-group associations between BOLD activation and the cognitive performance index (CPI). Statistical parametric maps were achieved using a mixed-effects model corrected for multiple comparisons using a cluster threshold of $\mathrm{Z}>2.3$ and a corrected cluster significance threshold of $p<0.05$. BOLD, blood oxygen level dependent; TBI, traumatic brain injury.

sample sizes ranging from 5 to 20 (median, 9) participants. ${ }^{8}$ Larger individual studies are needed, but multi-site collaborative initiatives combining data sets in meta-analyses should also be encouraged to gain critical new knowledge of functional brain adaptations after pediatric msTBI.

In conclusion, children and adolescents with slow IHTT after msTBI exhibit functional brain adaptations expressed as WMLdependent BOLD hyperactivations. Our previous studies in pediatric msTBI have also shown that slow IHTT is associated with widespread white matter disorganization in the post-acute phase, ${ }^{13}$ as well as prolonged neurodegeneration into the chronic phase. ${ }^{11,12}$ The combination of poor white matter organization, ${ }^{13}$ local structural hyperconnectivity, ${ }^{30}$ and slow IHTT $^{10}$ in a subset of children with msTBI may result in the need to recruit a broader network of neurons (i.e., increased BOLD activation) to support cognitive processes that require spatially distributed higher-order, capacitydemanding cognitive activities because of inefficiencies in recruiting more distal processing modules.

Our future analyses will use resting-state fMRI to directly evaluate the impact of poor white matter organization and reduced
IHTT on both local and global functional connectivity. Our current results represent a significant step toward a more substantial understanding of the effect of msTBI on the BOLD signal, and the link between hyperactivations and slow IHTT adds to the multi-modal validation of this electrophysiological measure as a promising biomarker. $^{14}$

\section{Acknowledgments}

We thank Leila Molina, Alma Martinez, and Alma Ramirez for their contribution to participant recruitment and study coordination and Edward Lau for assistance in implementing the fMRI task in Matlab. We also thank the families who participated in this study.

\section{Funding Information}

This research was supported by NICHD grant \#HD061504, Today's and Tomorrow's Children Fund, UCLA Brain Injury Research Center, and the Della Martin Foundation. P.T. was supported, in part, by NIH grants U54 EB020403 (from the NIH Big Data to Knowledge Program). 


\section{Author Disclosure Statement}

P.T. has received partial research support from Biogen, Inc. (Boston) for research not related to topics of this article.

\section{References}

1. Hillary, F.G. (2008). Neuroimaging of working memory dysfunction and the dilemma with brain reorganization hypotheses. J. Int. Neuropsychol. Soc. 14, 526-534.

2. Hillary, F.G., Genova, H.M., Chiaravalloti, N.D., Rypma, B., and DeLuca, J. (2006). Prefrontal modulation of working memory performance in brain injury and disease. Hum. Brain Mapp. 27, 837847.

3. Scheibel, R.S. (2017). Functional magnetic resonance imaging of cognitive control following traumatic brain injury. Front. Neurol. 8, 352 .

4. Hillary, F.G., and Biswal, B. (2007). The influence of neuropathology on the FMRI signal: a measurement of brain or vein? Clin. Neuropsychol. 21, 58-72.

5. Newsome, M.R., Steinberg, J.L., Scheibel, R.S., Troyanskaya, M., Chu, Z., Hanten, G., Lu, H., Lane, S., Lin, X., Hunter, J.V., Vasquez, C., Zientz, J., Li, X., Wilde, E.A., and Levin, H.S. (2008). Effects of traumatic brain injury on working memory-related brain activation in adolescents. Neuropsychology 22, 419-425.

6. Karunanayaka, P.R., Holland, S.K., Yuan, W., Altaye, M., Jones, B.V., Michaud, L.J., Walz, N.C., and Wade, S.L. (2007). Neural substrate differences in language networks and associated languagerelated behavioral impairments in children with TBI: a preliminary fMRI investigation. NeuroRehabilitation 22, 355-369.

7. Newsome, M.R., Scheibel, R.S., Chu, Z., Hunter, J.V., Li, X., Wilde, E.A., Lu, H., Wang, Z.J., Lin, X., Steinberg, J.L., Vasquez, A.C., Cook, L., and Levin, H.S. (2012). The relationship of resting cerebral blood flow and brain activation during a social cognition task in adolescents with chronic moderate to severe traumatic brain injury: a preliminary investigation. Int. J. Dev. Neurosci. 30, 255-266.

8. Dennis, E.L., Babikian, T., Giza, C.C., Thompson, P.M., and Asarnow, R.F. (2018). Neuroimaging of the injured pediatric brain: methods and new lessons. Neuroscientist 24, 652-670.

9. Cazalis, F., Babikian, T., Giza, C., Copeland, S., Hovda, D., and Asarnow, R.F. (2011). Pivotal role of anterior cingulate cortex in working memory after traumatic brain injury in youth. Front. Neurol. 1,158 .

10. Ellis, M.U., DeBoard Marion, S., McArthur, D.L., Babikian, T., Giza, C., Kernan, C.L., Newman, N., Moran, L., Akarakian, R., Houshiarnejad, A., Mink, R., Johnson, J., Babbitt, C.J., Olsen, A., and Asarnow, R.F. (2016). The UCLA study of children with moderate-tosevere traumatic brain injury: event-related potential measure of interhemispheric transfer time. J. Neurotrauma 33, 990-996.

11. Dennis, E.L., Faskowitz, J., Rashid, F., Babikian, T., Mink, R., Babbitt, C., Johnson, J., Giza, C.C., Jahanshad, N., Thompson, P.M., and Asarnow, R.F. (2017). Diverging volumetric trajectories following pediatric traumatic brain injury. Neuroimage Clin. 15, 125-135.

12. Dennis, E.L., Rashid, F., Ellis, M.U., Babikian, T., Vlasova, R.M., Villalon-Reina, J.E., Jin, Y., Olsen, A., Mink, R., Babbitt, C., Johnson, J., Giza, C.C., Thompson, P.M., and Asarnow, R.F. (2017). Diverging white matter trajectories in children after traumatic brain injury: the RAPBI study. Neurology 88, 1392-1399.

13. Dennis, E.L., Ellis, M.U., Marion, S.D., Jin, Y., Moran, L., Olsen, A., Kernan, C., Babikian, T., Mink, R., Babbitt, C., Johnson, J., Giza, C.C., Thompson, P.M., and Asarnow, R.F. (2015). Callosal function in pediatric traumatic brain injury linked to disrupted white matter integrity. J. Neurosci. 35, 10202-10211.

14. Guerriero, R.M., and Schlaggar, B.L. (2017). An important step toward a functional biomarker in pediatric TBI recovery and outcome. Neurology 88, 1386-1387.

15. Brunner, J.F., Olsen, A., Aasen, I.E., Lohaugen, G.C., Haberg, A.K., and Kropotov, J. (2015). Neuropsychological parameters indexing executive processes are associated with independent components of ERPs. Neuropsychologia 66, 144-156.

16. Hillary, F.G., Genova, H.M., Medaglia, J.D., Fitzpatrick, N.M., Chiou, K.S., Wardecker, B.M., Franklin, R.G., Jr., Wang, J., and DeLuca, J. (2010). The nature of processing speed deficits in traumatic brain injury: is less brain more? Brain Imaging Behav. 4, 141-154.
17. Rypma, B., Berger, J.S., Prabhakaran, V., Bly, B.M., Kimberg, D.Y., Biswal, B.B., and D'Esposito, M. (2006). Neural correlates of cognitive efficiency. Neuroimage 33, 969-979.

18. Banich, M.T., and Belger, A. (1990). Interhemispheric interaction: how do the hemispheres divide and conquer a task? Cortex 26, 77-94.

19. Price, C.J., Crinion, J., and Friston, K.J. (2006). Design and analysis of fMRI studies with neurologically impaired patients. J. Magn. Reson. Imaging 23, 816-826.

20. Moran, L.M., Babikian, T., Del Piero, L., Ellis, M.U., Kernan, C.L., Newman, N., Giza, C.C., Mink, R., Johnson, J., Babbitt, C., and Asarnow, R. (2016). The UCLA study of predictors of cognitive functioning following moderate/severe pediatric traumatic brain injury. J. Int. Neuropsychol. Soc. 22, 512-519.

21. Smith, S.M. (2002). Fast robust automated brain extraction. Hum. Brain Mapp. 17, 143-155.

22. Jenkinson, M., Bannister, P., Brady, M., and Smith, S. (2002). Improved optimization for the robust and accurate linear registration and motion correction of brain images. Neuroimage 17, 825-841.

23. Jenkinson, M., and Smith, S. (2001). A global optimisation method for robust affine registration of brain images. Med. Image Anal. 5, 143156.

24. Gosselin, N., Bottari, C., Chen, J.K., Petrides, M., Tinawi, S., De Guise, E., and Ptito, A. (2010). Electrophysiology and functional MRI reveal cerebral dysfunction after mild traumatic brain injury. Brain Inj. 24, 335-336.

25. Brown, W.S., Jeeves, M.A., Dietrich, R., and Burnison, D.S. (1999). Bilateral field advantage and evoked potential interhemispheric transmission in commissurotomy and callosal agenesis. Neuropsychologia 37, 1165-1180.

26. Nakamura, T., Hillary, F.G., and Biswal, B.B. (2009). Resting network plasticity following brain injury. PLoS One 4, e8220.

27. Pandit, A.S., Expert, P., Lambiotte, R., Bonnelle, V., Leech, R., Turkheimer, F.E., and Sharp, D.J. (2013). Traumatic brain injury impairs small-world topology. Neurology 80, 1826-1833.

28. Hillary, F.G., Rajtmajer, S.M., Roman, C.A., Medaglia, J.D., Slocomb-Dluzen, J.E., Calhoun, V.D., Good, D.C., and Wylie, G.R. (2014). The rich get richer: brain injury elicits hyperconnectivity in core subnetworks. PLoS One 9, e104021.

29. van den Heuvel, M.P., Kahn, R.S., Goni, J., and Sporns, O. (2012). High-cost, high-capacity backbone for global brain communication. Proc. Natl. Acad. Sci. U. S. A. 109, 11372-11377.

30. Dennis, E.L., Rashid, F., Babikian, T., Mink, R., Babbitt, C., Johnson, J., Giza, C.C., Asarnow, R.F., and Thompson, P.M. (2017). Altered network topology in pediatric traumatic brain injury. Presented at the 13th International Symposium on Medical Information Processing and Analysis, SPIE, San Andres Island, Colombia.

31. Hill, C.S., Coleman, M.P., and Menon, D.K. (2016). Traumatic axonal injury: mechanisms and translational opportunities. Trends Neurosci. $39,311-324$.

32. Strazzer, S., Rocca, M.A., Molteni, E., De Meo, E., Recla, M., Valsasina, P., Arrigoni, F., Galbiati, S., Bardoni, A., and Filippi, M. (2015). Altered recruitment of the attention network is associated with disability and cognitive impairment in pediatric patients with acquired brain injury. Neural Plast. 2015, 104282.

33. Babikian, T., and Asarnow, R. (2009). Neurocognitive outcomes and recovery after pediatric TBI: meta-analytic review of the literature. Neuropsychology 23, 283-296.

34. Sanchez-Carrion, R., Fernandez-Espejo, D., Junque, C., Falcon, C., Bargallo, N., Roig, T., Bernabeu, M., Tormos, J.M., and Vendrell, P. (2008). A longitudinal fMRI study of working memory in severe TBI patients with diffuse axonal injury. Neuroimage $43,421-429$.

35. Lieberman, M.D., and Cunningham, W.A. (2009). Type I and type II error concerns in fMRI research: re-balancing the scale. Soc. Cogn. Affect. Neurosci. 4, 423-428.

Address correspondence to: Alexander Olsen, PhD

Department of Psychology

Norwegian University of Science and Technology 7491 Trondheim Norway

E-mail: alexander.olsen@ntnu.no 